\title{
Nano-bio interactions between carbon nanomaterials and blood plasma proteins: why oxygen functionality matters
}

\begin{abstract}
Kenry ${ }^{1,2,3}$, Alisha Geldert ${ }^{3}$, Yanpeng Liu ${ }^{2,4}$, Kian Ping Loh ${ }^{1,2,4}$ and Chwee Teck Lim ${ }^{1,2,3,5}$
Carbon nanomaterials are some of the most versatile nanomaterials. Along with increasing explorations into their utilization in a plethora of biological and biomedical applications, there have been emerging interests and needs in understanding the molecular hemocompatibility of these engineered nanomaterials when coming into contact with blood. Here, we evaluate the nano-bio interactions of one-dimensional (1D) and two-dimensional (2D) carbon nanomaterials with blood plasma proteins. Different facets of the nanomaterial-protein interactions, specifically, the adsorption, equilibrium binding and conformational stability of proteins upon association with carbon nanomaterials are established, based on the quantification of various parameters, such as association constant, binding cooperativity and protein secondary structural change. In light of our data, we demonstrate that the carbon nanomaterial-plasma protein interactions may be significantly influenced by the density of the oxygenated functionalities of the nanomaterials and to a certain extent, their dimensionality and surface area. This work offers a broad insight into the nano-bio interactions between carbon nanomaterials and blood plasma proteins and provides a strong basis for the design and use of $1 \mathrm{D}$ and $2 \mathrm{D}$ carbon nanomaterials for a wide variety of bioapplications.
\end{abstract}

NPG Asia Materials (2017) 9, e422; doi:10.1038/am.2017.129; published online 18 August 2017

\section{INTRODUCTION}

The exploration into the potential uses of carbon nanomaterials, particularly one-dimensional (1D) carbon nanotubes $(\mathrm{CNTs})^{1-3}$ and two-dimensional (2D) graphene, ${ }^{4-7}$ in a wide spectrum of biological and biomedical applications, ${ }^{8-19}$ has led to an immediate need to elucidate their pharmacological and toxicological profiles. ${ }^{20-23}$ In particular, the assessment of the hemocompatiblity of these engineered nanomaterials based on their interactions with blood components has attracted much attention in recent years. ${ }^{16-18}$ This is because, for most of the in vivo bioapplications of carbon nanomaterials, there is likelihood of them being exposed to blood cells and plasma proteins in the blood circulatory system. As carbon nanomaterials enter a physiological environment, they will inevitably interact and bind with specific proteins in that microenvironment. This binding is normally influenced by several factors, notably the nanomaterial surface properties. ${ }^{24-26}$ Moreover, the nanomaterial-protein association significantly influences the biological effects of carbon nanomaterials as well as the extent of the biological response towards these nanomaterials. ${ }^{27-29}$ Therefore, comprehensive investigations on the characteristics of the nanomaterial-protein binding in biological fluids are necessary for elucidating the influence and physiological effects of the carbon nanomaterials.
In general, biological fluids such as blood plasma comprise many crucial proteins, including albumin, globulin and fibrinogen, possessing unique and distinct functionalities. ${ }^{30,31}$ As one of the smaller biomolecules with a molecular size of approximately $6.1 \times 8.5 \times 7 \mathrm{~nm}$, albumin plays a crucial part in the physiological transport of numerous biomolecules. It is the most abundant protein in the blood plasma. Globulin, on the other hand, is the second most abundant plasma protein responsible for body immune system and molecular transport. It has a molecular size of about $13.7-27.4 \times 16.6 \times 6.3 \mathrm{~nm}$. In contrast, as the largest blood plasma protein with a molecular size of roughly $25.3 \times 6.5 \times 53.5 \mathrm{~nm}$, fibrinogen plays a critical part in the blood coagulation process in mediating the production of fibrin and binding of platelets.

Recent years have seen increasing studies on the associations between carbon nanomaterials and proteins as well as the biological effects induced by these nanomaterials. ${ }^{16,31,32}$ Nonetheless, most of these studies have been performed on 1D CNTs and limited attempts have been made on 2D graphene-based nanomaterials. Furthermore, the effects of the density of oxygenated functional groups and dimensionality of carbon nanomaterials on the nanomaterial-protein interactions have not been well elucidated. As such, here, we seek to elucidate the interactions between carbon nanomaterials with different

\footnotetext{
${ }^{1}$ NUS Graduate School for Integrative Sciences and Engineering, National University of Singapore, Singapore, Singapore; ${ }^{2}$ Centre for Advanced $2 \mathrm{D}$ Materials and Graphene Research Centre, National University of Singapore, Singapore, Singapore; ${ }^{3}$ Department of Biomedical Engineering, National University of Singapore, Singapore, Singapore; ${ }^{4}$ Department of Chemistry, National University of Singapore, Singapore, Singapore and ${ }^{5}$ Mechanobiology Institute, National University of Singapore, Singapore, Singapore Correspondence: Professor CT Lim, Department of Biomedical Engineering, National University of Singapore, 9 Engineering Drive 1, Singapore 117576, Singapore. E-mail: ctlim@nus.edu.sg Received 21 October 2016; revised 30 March 2017; accepted 1 June 2017
} 
oxygenated group densities and dimensionalities and blood plasma proteins. Specifically, three distinct carbon nanomaterials, that is, 1D carboxylic-functionalized multiwalled CNT (CNT-COOH) as well as 2D graphene nanoplatelets (GNPs) and porous graphene oxide (PGO), and three major blood plasma proteins, that is, albumin, globulin and fibrinogen, were used in this study (Scheme 1). We probe various aspects of the interactions, in particular, the adsorption, the binding kinetics and equilibrium, and the secondary structural stability of plasma proteins in the presence of those carbon nanomaterials. In light of the observations from this study, we anticipate that the effects of the density of the oxygenated functional groups of carbon nanomaterials as well as their dimensionality on their interactions with blood plasma proteins would be unveiled.

\section{MATERIALS AND METHODS}

\section{Materials}

CNT-COOH and GNPs were used directly without further purification (Cheaptubes, Inc., Cambridgeport, VT, USA). PGO was prepared based on the sequential acid and base treatments of GO synthesized through Hummer's method. ${ }^{33}$ All carbon nanomaterials in suspensions were prepared in MilliQ water. All plasma protein powders (Sigma-Aldrich, St Louis, MO, USA), that is, albumin (A9511), globulin (G4386) and fibrinogen (F4883), were dissolved in phosphate-buffered saline $(1 \times)$.

\section{Nanomaterial and protein morphological and optical property characterization}

Carbon nanomaterial, plasma protein, and nanomaterial-protein complex samples were initially deposited on mica, gently washed and air-dried. Their surface morphology was examined utilizing the tapping mode atomic force microscope (AFM) (Bruker, Billerica, MA, USA). The Raman spectra of carbon nanomaterials were collected using a $0.03 \mathrm{~mW}$ WITec Alfa 300R. Excitation was set at $532 \mathrm{~nm}$ at an integration time of $2 \mathrm{~s}$ over 10 accumulations. The Fourier Transform Infrared (FTIR) spectra of carbon nanomaterials were obtained using a Bio-Rad FTS-3500 ARX FTIR spectrometer operating in transmission mode. The X-ray photoelectron spectroscopy (XPS) spectra of carbon nanomaterials were collected using SPECS XR-50X-ray Mg K $\alpha$ $(1253.7 \mathrm{eV})$ source with a pass energy of $30 \mathrm{eV}$ and a spot size of $5 \mathrm{~mm}$ in an ultrahigh vacuum chamber (SPECS, base pressure of $1 \times 10^{-10}$ Torr). The detection was carried out using a PHOIBOS 150 hemispherical energy analyzer (SPECS, GmbH, Berlin, Germany) equipped with a 3D delay line

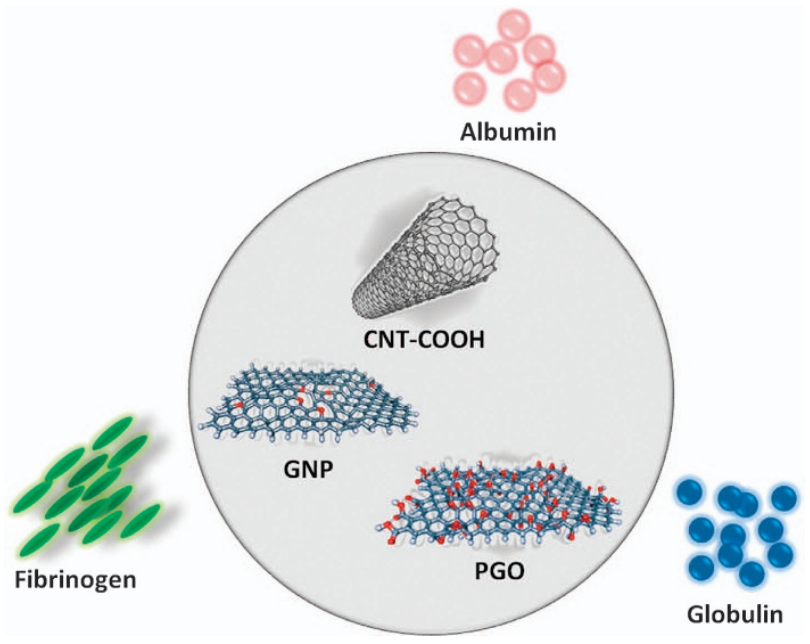

Scheme 1 Nano-bio interactions between carbon nanomaterials and blood plasma proteins. 1D CNT-COOH and 2D GNP and PGO with different dimensionalities, surface areas and oxygen functionality densities interact with albumin, globulin and fibrinogen. detector (3D-DLD, SPECS GmbH). The calibration of the binding energies of the XPS spectra was performed with respect to the Au $4 f_{7 / 2}$ peaks. The fitting of the XPS peaks was performed based on a mixed Gaussian-Lorentzian function post a Shirley background subtraction. The absorption spectra of carbon nanomaterials and plasma proteins were acquired utilizing an ultraviolet-visible (UV-Vis) spectrophotometer (NanoDrop 2000, Thermo Scientific, Waltham, MA, USA). The intrinsic fluorescence spectra of plasma proteins were recorded using a microplate reader (Infinite M200, Tecan Trading AG, Mannedorf, Switzerland). Excitation was fixed at $280 \mathrm{~nm}$ while emission was measured from 300 to $450 \mathrm{~nm}$.

\section{Absorbance characterization}

All plasma proteins were first prepared at various concentrations of 1, 2, 5, 10 and $20 \mathrm{mg} \mathrm{ml}^{-1}$ for free protein absorbance measurements. Next, all carbon nanomaterials were fixed at $100 \mu \mathrm{g} \mathrm{ml}^{-1}$ while the plasma proteins were prepared at numerous concentrations of $0.5,1,2.5$ and $5 \mathrm{mg} \mathrm{ml}^{-1}$ for the evaluation of the adsorption of plasma proteins on carbon nanomaterials. Carbon nanomaterials $(30 \mu \mathrm{l})$ were subsequently mixed with plasma proteins $(30 \mu \mathrm{l})$ and incubated for $10 \mathrm{~min}$ at room temperature. The protein-nanomaterial mixtures were then centrifuged at $13000 \mathrm{~g}$ for $7 \mathrm{~min}$ and the supernatant was acquired for the measurements of protein adsorption isotherm at $280 \mathrm{~nm}$. The amount of proteins adsorbed on carbon nanomaterials was taken as the difference in the protein absorbance in the absence and presence of nanomaterials. All reported constants were acquired as the average of three independent trials performed at room temperature. The obtained adsorption data were subsequently fitted based on the Langmuir-Freundlich adsorption isotherm.

\section{Fluorescence quenching characterization}

Varying concentrations of each nanomaterial solution were mixed with a fixed concentration of plasma protein in black microplate wells (Nunc MicroWell, Thermo Scientific) to yield a total volume of $200 \mu \mathrm{l}$ in each well and a final plasma protein concentration of $500 \mathrm{\mu g} \mathrm{m}^{-1}$. Carbon nanomaterials were prepared from $1 \mu \mathrm{g} \mathrm{ml}^{-1}$ to concentrations sufficient to achieve at least $80 \%$ fluorescence quenching efficiency. The Stern-Volmer constant $\left(K_{S V}\right)$ indicating the relative fluorescence quenching efficiency of carbon nanomaterials was calculated by taking the slope of the linear portion of the plot of $I^{0} / I$ vs nanomaterial concentration, based on non-equilibrium Stern-Volmer equation, as illustrated in Equation (1),

$$
\frac{I^{0}}{I}=1+K_{\mathrm{SV}}[\text { Nanomaterial }]
$$

where $I^{0}$ is the peak fluorescence emission of pure plasma proteins, $I$ is the peak fluorescence emission of the nanomaterial-protein complexes and [Nanomaterial] is the concentration of carbon nanomaterials. Quenching ratio $(Q)$ at each nanomaterial concentration was calculated as

$$
Q=\frac{\left(I^{0}-I\right)}{I^{0}}
$$

The equilibrium dissociation constant $\left(K_{\mathrm{D}}\right)$ and the Hill coefficient $(n)$ measuring the relative strength and cooperativity of the nanomaterial-protein bindings, respectively, were derived by fitting the quantitative data to the Hill equation, as illustrated in Equation (3), based on nonlinear least-squares regression.

$$
\frac{Q}{Q_{\max }}=\frac{[\text { Nanomaterial }]^{n}}{K_{\mathrm{D}}^{n}+[\text { Nanomaterial }]^{n}}
$$

where $Q_{\max }$ is the saturation value of the quenching ratio. All reported constants were acquired as the average of three independent trials performed at room temperature.

\section{Circular dichroism characterization}

Conformational stability of plasma proteins pre- and post-incubations with carbon nanomaterials was evaluated using circular dichroism (CD) spectroscopy (Jasco J-810). Albumin, globulin and fibrinogen were prepared at fixed concentrations of 200, 400 and $400 \mu \mathrm{g} \mathrm{ml}^{-1}$, correspondingly. Carbon 
nanomaterials were prepared such that the protein:nanomaterial concentration ratios were fixed at 1:0.025, 1:0.05 and 1:0.1. A 1-mm quartz cell was used for all CD measurements. Scanning speed and wavelength range were fixed at $50 \mathrm{~nm}$ per min and 200-260 nm, respectively. All CD spectra were acquired at room temperature as an accumulation of three continuous scans.

\section{Statistical analysis}

All quantitative data were represented as mean \pm s.d. The evaluation of the statistical differences between experimental data was performed based on the Student's $t$-test. Statistically significant difference was defined by ${ }^{*}$ for $P<0.05$ (that is, 95\% confidence intervals) and ${ }^{\star *}$ for $P<0.01$ (that is, 99\% confidence intervals).

\section{RESULTS AND DISCUSSION}

Morphological and optical characterization of carbon nanomaterials and proteins

We first began characterizing the morphological features of carbon nanomaterials and plasma proteins (Figure 1). Aqueous suspensions of different carbon nanomaterial and plasma protein samples were first dropped on mica and their morphology were probed using an AFM operating in the tapping mode. Based on the obtained data, we observed that the three carbon nanomaterials of CNT-COOH, GNP and PGO possessed distinct surface morphologies and structures. CNT-COOH displayed a short tubular structure with a width of about $80 \mathrm{~nm}$ and a height of roughly 12-16 nm (Figure 1a). GNP displayed an irregular shape coupled with a flat surface morphology, with a height of $10 \mathrm{~nm}$ (Figure 1b). PGO, in contrast, exhibited a wrinkled morphological flat structure filled with pores and surface defects. Also, it had a height of between 2 and $4 \mathrm{~nm}$ (Figure 1c). The three plasma proteins, on the other hand, possessed highly regular surface morphology and physical size of between a few to tens of $\mathrm{nm}$. More specifically, the smaller albumin and globulin molecules had a size of a few nm (Figures 1d and e) while the fibrinogen molecules were slightly larger with a size in terms of tens of nm (Figure 1f).

Subsequent to examining the morphological features of individual carbon nanomaterials and plasma proteins, we proceeded to characterize the surface morphology of various nanomaterial-protein complexes (Figure 2). Interestingly, for all complexes, the formation of protein aggregates on the nanomaterial surfaces was evident. Grainier and rougher topographical features could also be observed on the surface of the complexes as compared to that of bare carbon nanomaterials. For example, the surface of bare GNP was relatively flat and smooth while those of its complexes were rough and filled with grainy particles of various sizes and distributions (Figures $2 \mathrm{~d}-\mathrm{f}$ ). In fact, similar irregular topographical features were manifested by the complexes of CNT-COOH (Figure 2a-c) and PGO (Figures 2g-i).

Following the surface morphological characterization, we sought to probe the optical properties of carbon nanomaterials and blood plasma proteins (Figure 3). First, the crystal quality of carbon nanomaterials were revealed using Raman spectroscopy (Figure 3a). The acquired Raman spectrum of CNT-COOH demonstrated strong $D$ and $G$ peaks comparable in their signal intensity at 1332 and $1569 \mathrm{~cm}^{-1}$, respectively. This signifies a high defect concentration of CNT-COOH. A strong 2D peak at $2675 \mathrm{~cm}^{-1}$ coupled with a weak $\mathrm{D}+\mathrm{G}$ band at $2925 \mathrm{~cm}^{-1}$ could also be determined from the Raman spectrum of CNT-COOH. GNP, meanwhile, had a Raman spectrum showing a weak defect-associated D peak and a strong $G$ peak at 1332 and $1561 \mathrm{~cm}^{-1}$, correspondingly. The $\mathrm{G}$ peak had a significantly higher relative intensity than the $\mathrm{D}$ peak, suggesting a low defect concentration of GNP. A distinct 2D band was also noted at the high wavenumber of $2685 \mathrm{~cm}^{-1}$. In contrast to CNT-COOH and GNP, strong and broad D and G bands at roughly 1332 and $1589 \mathrm{~cm}^{-1}$, correspondingly, were evident in the Raman spectrum of PGO. In fact, the relative intensities of both D and G bands were similar, indicating that PGO possessed a high defect concentration and significant structural disorder. Interestingly, the D and G peaks of PGO were much broader than those of CNT-COOH. This translates into the possibility that $\mathrm{PGO}$ might have much more defect variations as compared to CNT-COOH. The Raman spectrum of PGO also revealed three weak and inseparable bands (that is, $2 \mathrm{D}, \mathrm{D}+\mathrm{G}$, and $2 \mathrm{D}^{\prime}$ ) at high wavenumber beyond $2500 \mathrm{~cm}^{-1}$, further indicating a high concentration of defects. The optical absorbance of carbon nanomaterials was then characterized via UV-vis spectroscopy (Figure 3b). Specifically, both CNT-COOH and GNP did not exhibit any distinct absorption peak while the characteristic absorbance peak of PGO was observed at roughly $260 \mathrm{~nm}$.

Next, the functional elements of the different carbon nanomaterials were unveiled using FTIR spectroscopy under room temperature (Figures 3c-f). In light of the obtained infrared spectra of all nanomaterials (Figure 3c), we noted that the three carbon nanomaterials incorporated different oxygenated functional groups to a certain extent. For example, the infrared spectrum of CNT-COOH indicated several distinct peaks characteristic of oxygen functionalities (Figure 3d). Contributions from different oxygen functional groups, such as carboxyls (that is, $\mathrm{COOH}$ at roughly $1050-1150 \mathrm{~cm}^{-1}$, $1650-1750 \mathrm{~cm}^{-1}$, and $3300-3600 \mathrm{~cm}^{-1}$ ), carbonyls (that is, $\mathrm{C}=\mathrm{O}$ at about $1500-1750 \mathrm{~cm}^{-1}$ ), hydroxyls (that is, $\mathrm{C}-\mathrm{OH}$ at approximately $1050-1150 \mathrm{~cm}^{-1}$ and $3300-3600 \mathrm{~cm}^{-1}$ ) and ethers (that is, C-O at $800-1200 \mathrm{~cm}^{-1}$ ) were evident. At the same time, numerous peaks beyond $3600 \mathrm{~cm}^{-1}$ existed and these could be ascribed to the free hydroxyl groups. The FTIR spectrum of GNP also exhibited several peaks indicative of the presence of oxygenated groups (Figure 3e). In fact, most of the oxygen functionality-related peaks were less pronounced as compared to those of CNT-COOH, but they could still be observed from the infrared spectrum of GNP albeit with much lower intensity. Most importantly, the contribution of the $\mathrm{O}-\mathrm{H}$ stretching vibrations or oscillations of the carboxyl group, observable from the wavenumber between $3300 \mathrm{~cm}^{-1}$ and $3600 \mathrm{~cm}^{-1}$, was insignificant. This suggests the lower density of the oxygen functionalities possessed by GNP. PGO, in contrast, exhibited the most evident presence of the oxygenated functionality-related peaks in its FTIR spectrum (Figure 3f). In addition to carboxyls, carbonyls, hydroxyls and ethers, as evidenced from the existence of numerous peaks, notably those at $1035 \mathrm{~cm}^{-1}$ (that is, C-O-C stretching vibrations), $1234 \mathrm{~cm}^{-1}$ (that is, $\mathrm{C}-\mathrm{OH}$ stretching vibrations), $1597 \mathrm{~cm}^{-1}$ (that is, unoxidized graphitic domain skeletal vibrations) and $1705 \mathrm{~cm}^{-1}$ (that is, $\mathrm{C}=\mathrm{O}$ stretching vibrations), it was highly likely that PGO also possessed other oxygen-containing groups, such as epoxides (that is, $\mathrm{C}-\mathrm{O}-\mathrm{C}$ at $850 \mathrm{~cm}^{-1}$ and $1280-1330 \mathrm{~cm}^{-1}$ ), and $\mathrm{sp}^{2}$-hybridized $\mathrm{C}=\mathrm{C}$ (that is, $\mathrm{sp}^{2}-\mathrm{C}$, in-plane vibrations at $1597 \mathrm{~cm}^{-1}$ ).

To further quantify the elemental contents of the three carbon nanomaterials, their chemical environments were characterized using XPS (Figures 3g-1). Based on the elemental analysis, it was revealed that the three carbon nanomaterials consisted primarily of carbon and oxygen (Figures 3g-i). More clearly, for CNT-COOH, carbon constituted $85.47 \%$ while oxygen constituted $14.53 \%$ (Figure $3 g$ ). Its highresolution $\mathrm{C} 1 \mathrm{~s}$ spectrum showed that $\mathrm{CNT}-\mathrm{COOH}$ possessed $51.40 \%$ $\mathrm{C}-\mathrm{C}, 17.03 \% \mathrm{C}-\mathrm{O}$ and $17.02 \% \mathrm{C}=\mathrm{O}$ bonds (Figure $3 \mathrm{j}$ ). GNP possessed $90.13 \%$ carbon and $9.87 \%$ oxygen (Figure $3 \mathrm{~h}$ ), with its 
$\mathbf{a}_{\mathbf{i}}$
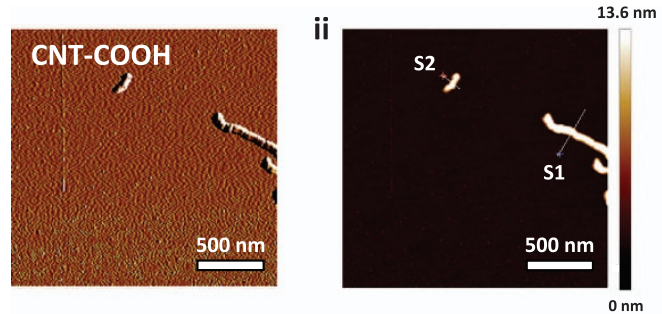

$\mathbf{b}_{\mathrm{i}}$

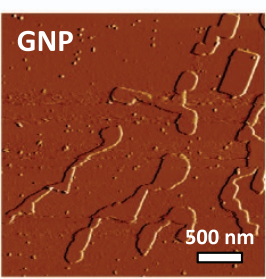

$\mathbf{C}_{\mathbf{i}}$

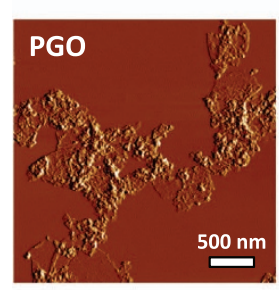

$\mathbf{d}_{\mathbf{i}}$

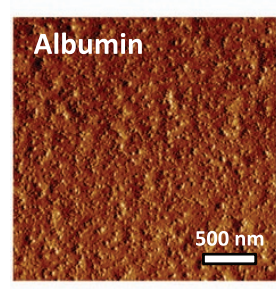

$\mathbf{e}_{\mathbf{i}}$

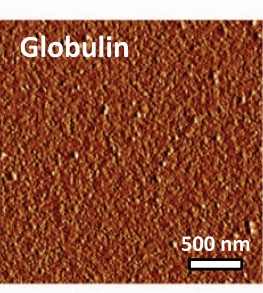

$\mathbf{f}_{\mathbf{i}}$

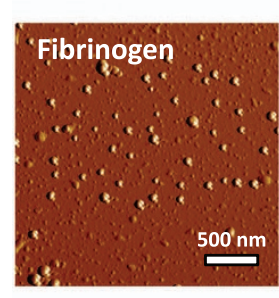

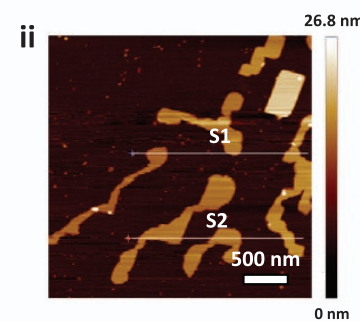
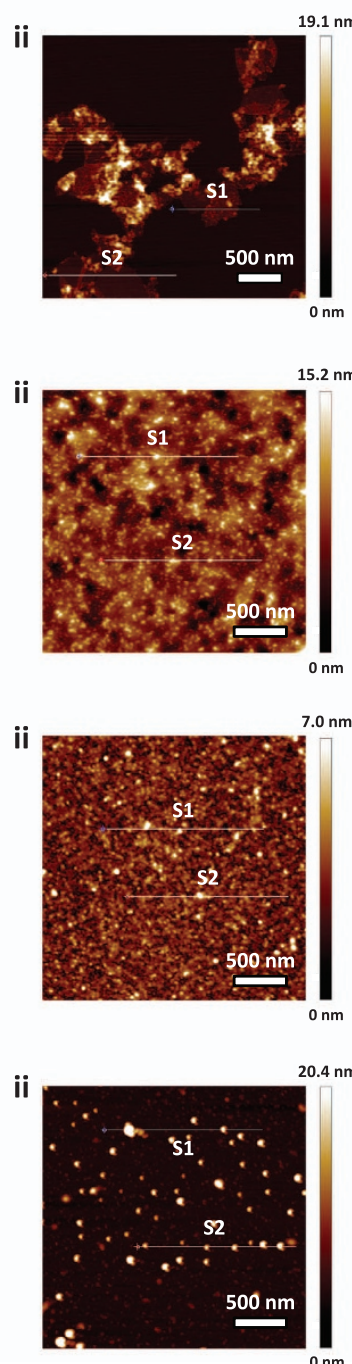
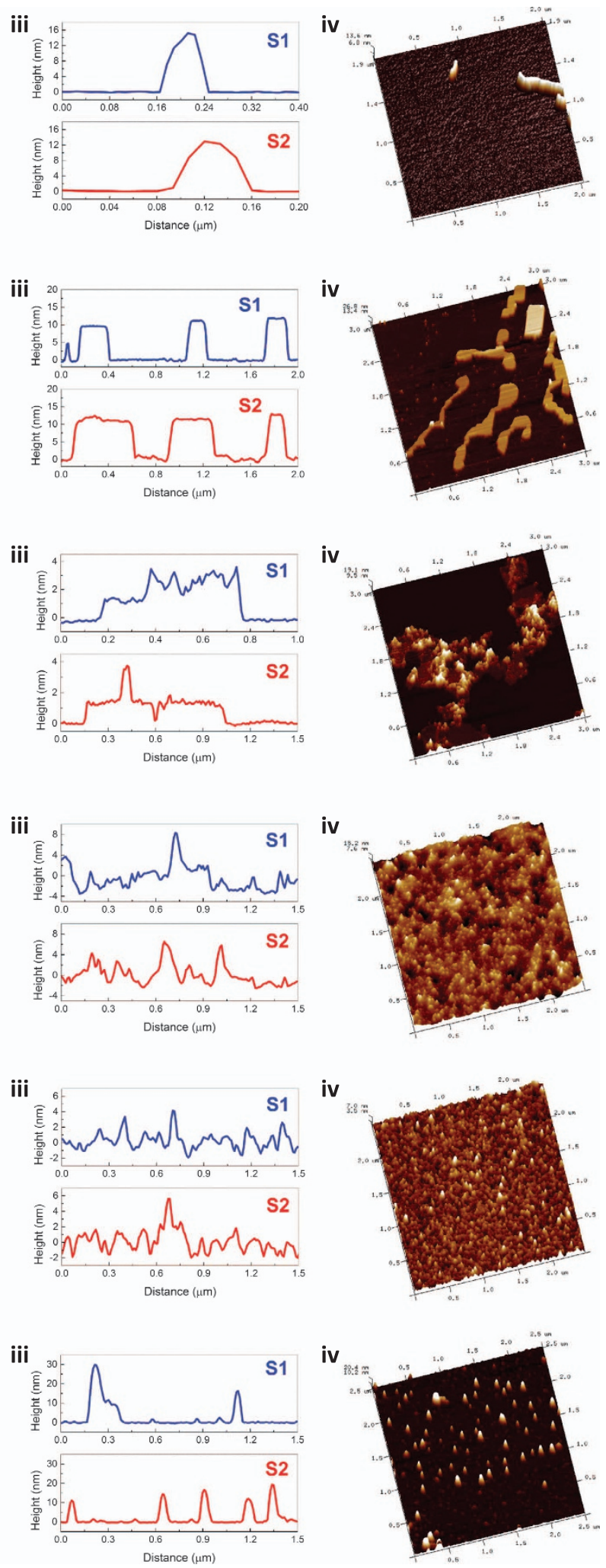

Figure 1 Surface morphology of carbon nanomaterials and blood plasma proteins. Representative AFM images of (a) CNT-COOH, (b) GNP, (c) PGO, (d) Albumin, (e) Globulin and (f) Fibrinogen, where: (i) Amplitude images of the nanomaterials and plasma proteins. (ii) 2D height images of the nanomaterials and plasma proteins with (iii) their respective sectional profiles (that is, S1 and S2 in Figure ii). (iv) 3D height images of the nanomaterials and plasma proteins. Scale bars represent $500 \mathrm{~nm}$. AFM, atomic force microscope; CNT-COOH, $\mathrm{COOH}$-functionalized carbon nanotube; GNP, graphene nanoplatelet; PGO, porous graphene oxide. 

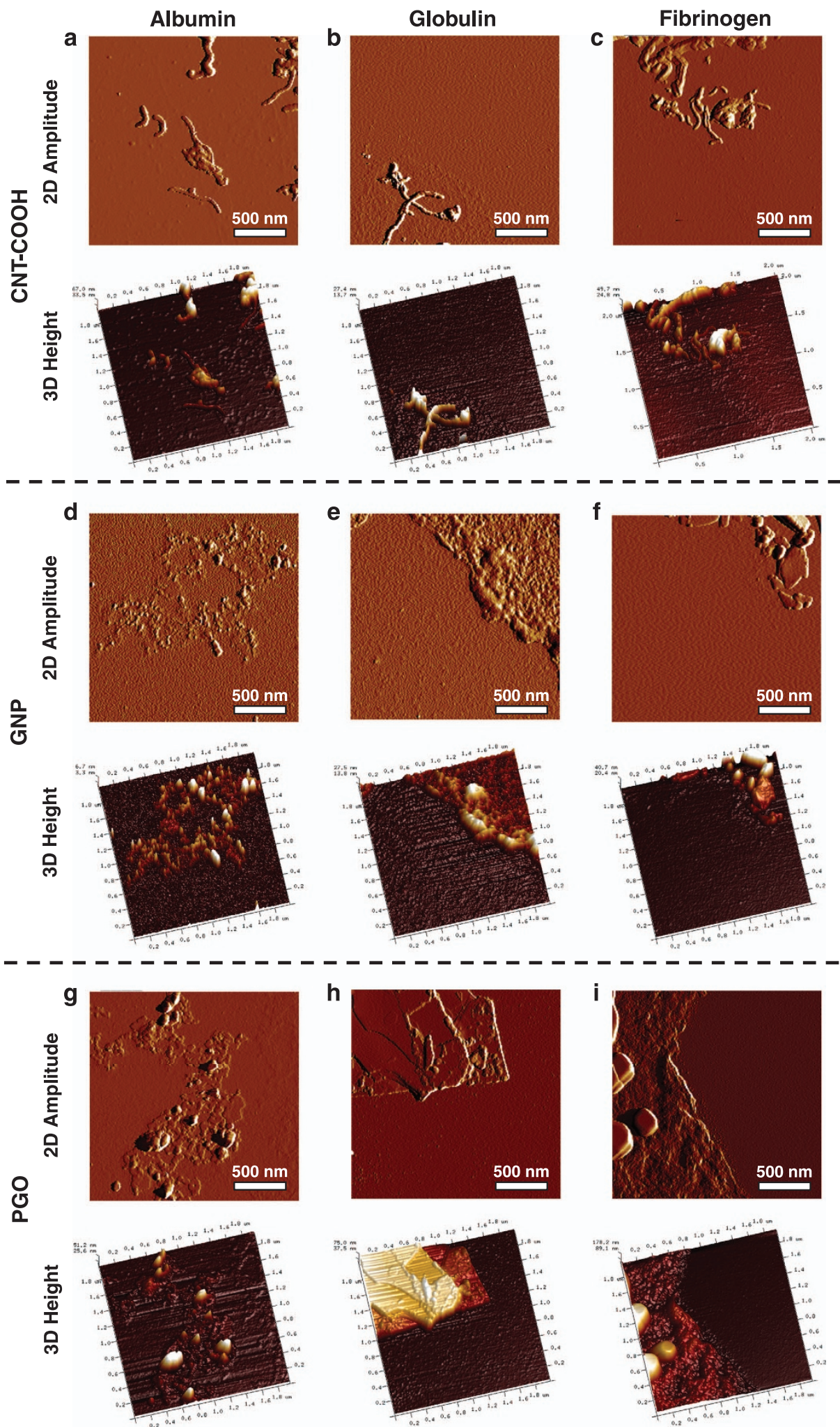

Figure 2 Surface morphology of the various nanomaterial-protein complexes. Representative 2D amplitude images (top) and 3D height images (bottom) of: (a) CNT-COOH-albumin, (b) CNT-COOH-globulin, (c) CNT-COOH-fibrinogen, (d) GNP-albumin, (e) GNP-globulin, (f) GNP-fibrinogen, (g) PGO-albumin, (h) PGO-globulin and (i) PGO-fibrinogen. Scale bars represent $500 \mathrm{~nm}$. CNT-COOH, COOH-functionalized carbon nanotube; GNP, graphene nanoplatelet; PGO, porous graphene oxide. 
a

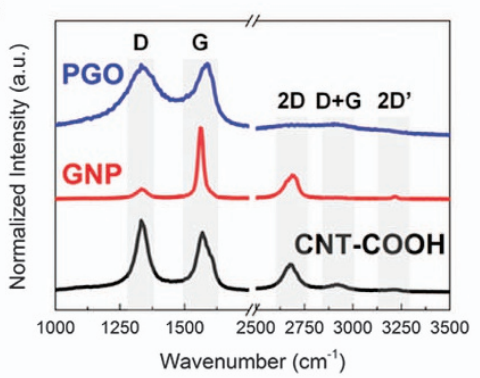

d

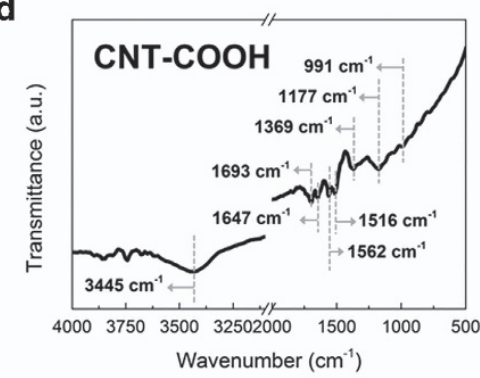

g

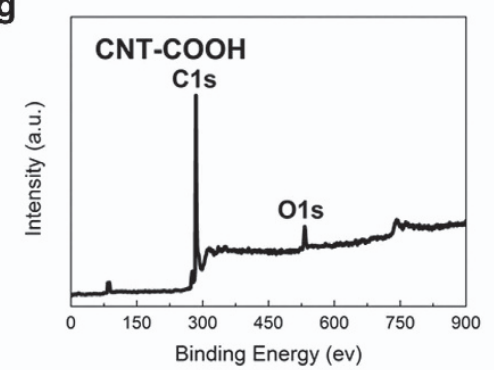

j
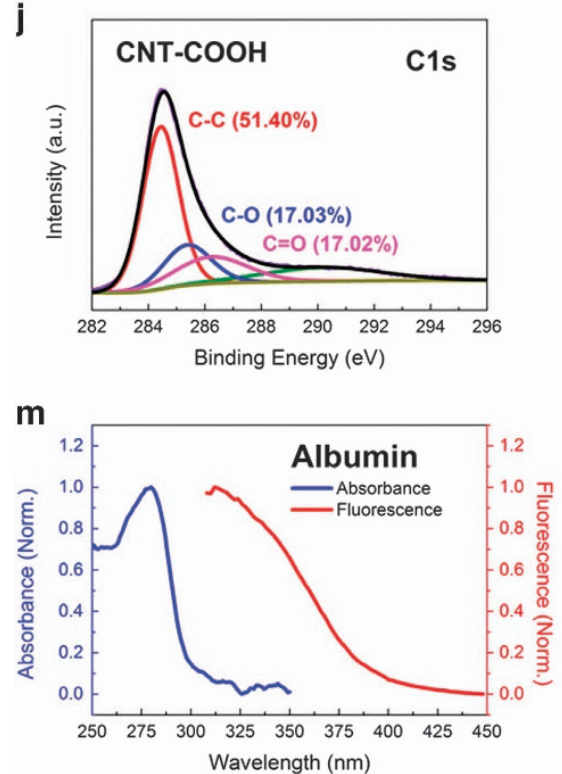

b

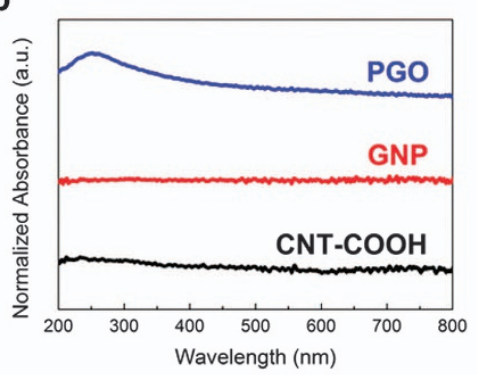

e

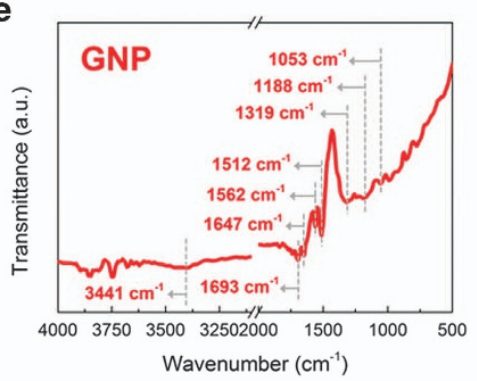

h

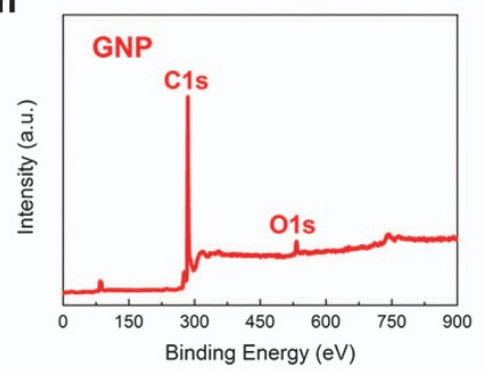

k

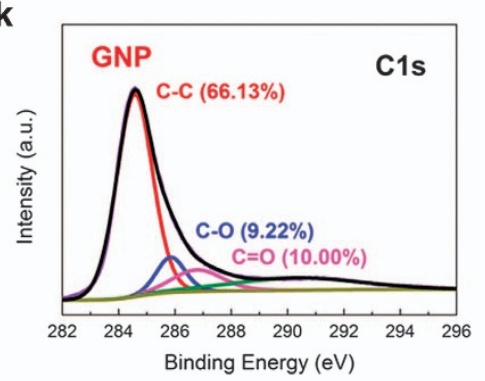

n

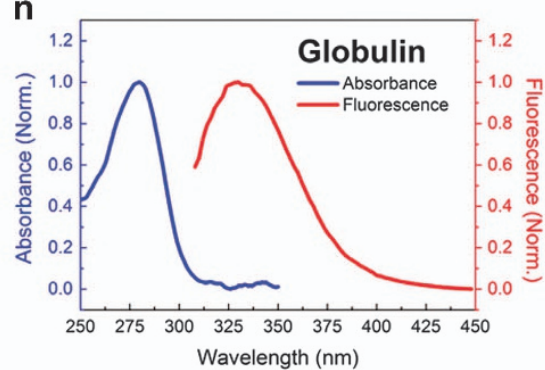

C

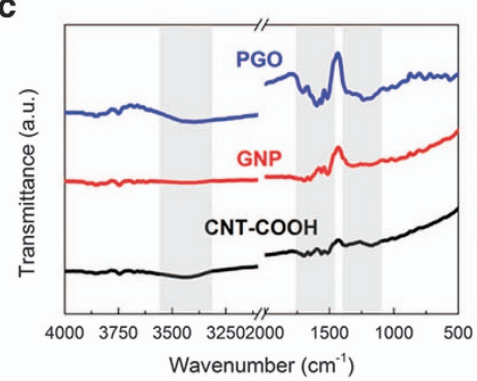

f

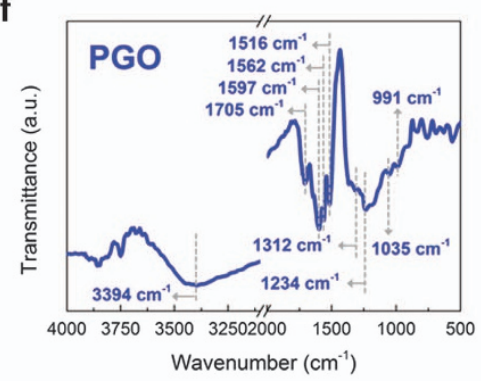

i

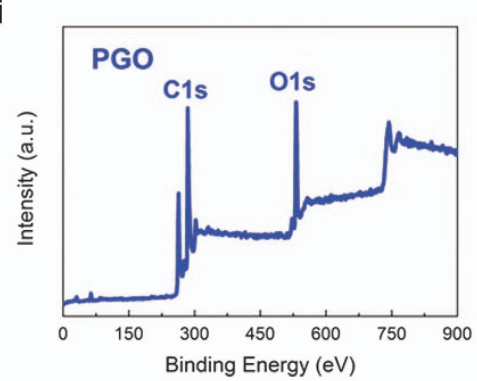

I

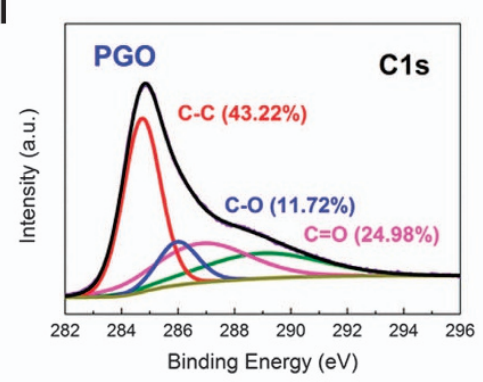

0

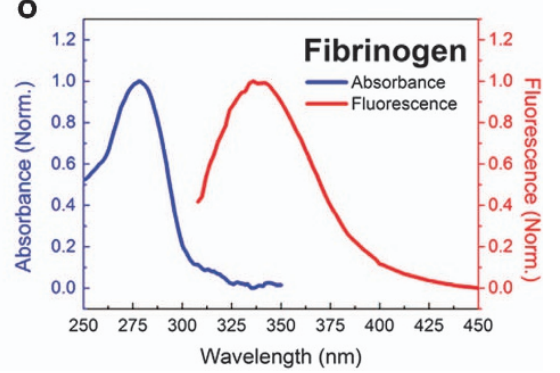

Figure 3 Optical properties of carbon nanomaterials and blood plasma proteins. (a-c) Optical spectra of all carbon nanomaterials: (a) Raman, (b) UV-vis and (c) FTIR. For FTIR spectra, the highlighted parts (in gray) represent the regions corresponding to various oxygenated functional elements, such as carbonyls, carboxyls, hydroxyls, ethers and epoxides. (d-f) Individual FTIR spectra of: (d) CNT-COOH, (e) GNP, (f) PGO, illustrating the various characteristic peaks corresponding to the oxygenated functional elements. (g-i) Wide scan XPS spectra of: (g) CNT-COOH, (h) GNP and (i) PGO, with (j-I) their corresponding C1s peaks showing the percentages of $\mathrm{C}-\mathrm{C}, \mathrm{C}-\mathrm{O}$ and $\mathrm{C}=\mathrm{O}$ bonds. ( $\mathbf{m}-\mathbf{0})$ Normalized UV-vis absorbance and fluorescence of the three blood plasma proteins: ( $\mathrm{m}$ ) albumin, (n) globulin and (o) fibrinogen. CNT-COOH, $\mathrm{COOH}$-functionalized carbon nanotube; FTIR, Fourier Transform Infrared; GNP, graphene nanoplatelet; PGO, porous graphene oxide; XPS, X-ray photoelectron spectroscopy. 
$\mathrm{C}-\mathrm{C}, \mathrm{C}-\mathrm{O}$ and $\mathrm{C}=\mathrm{O}$ bonds constituting $66.13,9.22$ and $10.00 \%$, respectively (Figure $3 \mathrm{k}$ ). The carbon and oxygen contents of PGO, on the other hand, were comparable (Figure 3i). In fact, the $\mathrm{C}-\mathrm{C}, \mathrm{C}-\mathrm{O}$ and $\mathrm{C}=\mathrm{O}$ bonds of $\mathrm{PGO}$ constituted 43.22, 11.72 and 24.98\%, respectively (Figure 31). Altogether, based on the FTIR and XPS data, we deduced that PGO possessed the highest density of oxygenated functionalities among the three carbon nanomaterials. GNP, in contrast, displayed the lowest density of oxygen functional elements. It is also interesting to note that the density of the oxygenated functional elements of carbon nanomaterials might be positively correlated to their concentrations of defects, as inferred from their Raman spectra.

The absorbance and intrinsic fluorescence emission of albumin, globulin and fibrinogen were also evaluated (Figures $3 \mathrm{~m}-\mathrm{o}$ ). It was
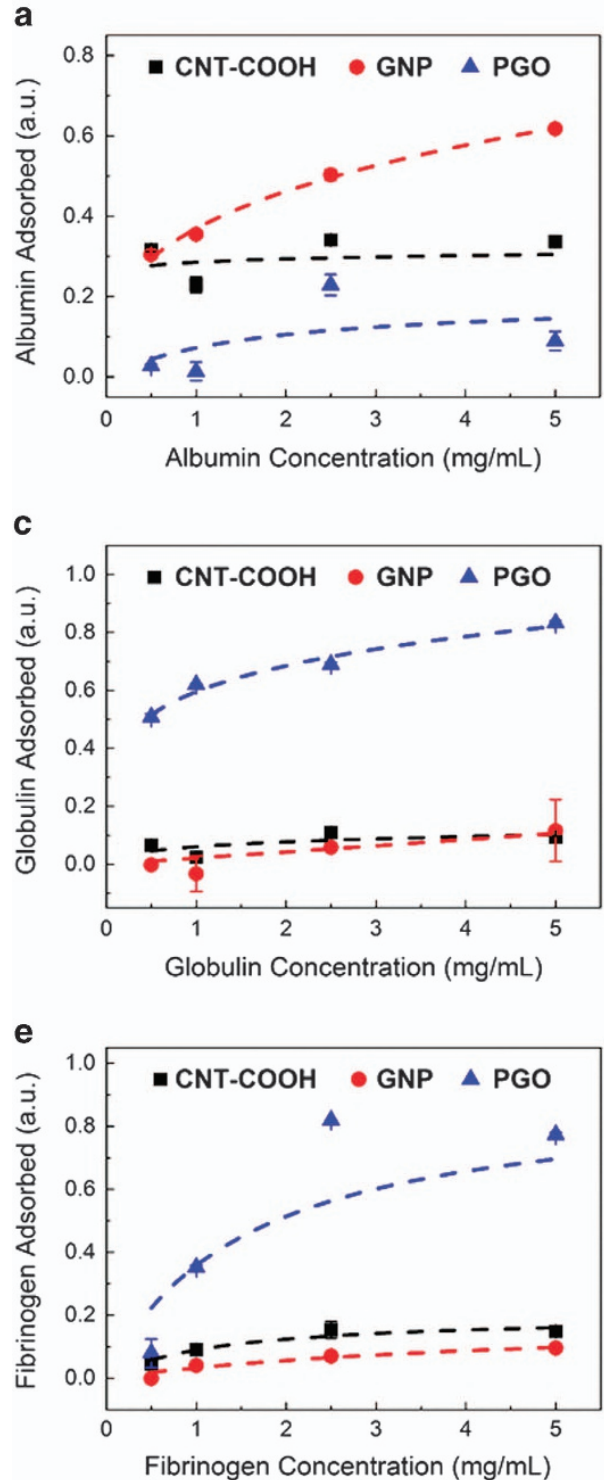

noted that the three plasma proteins exhibited a characteristic peak absorbance at about $280 \mathrm{~nm}$. In contrast, their peak fluorescence emission varied in which albumin, globulin and fibrinogen displayed peak intrinsic fluorescence at approximately 312, 330 and $336 \mathrm{~nm}$, respectively.

\section{Loading of plasma proteins on carbon nanomaterials}

To elucidate the nano-bio interactions between carbon nanomaterials and plasma proteins, we started probing the protein adsorption on carbon nanomaterials (Figure 4 and Supplementary Figure S1). Specifically, the loading capacity of plasma proteins on carbon nanomaterials was unveiled from the protein absorbance pre- and post-incubation with CNT-COOH, GNP and PGO, following the protocol reported in our previous study. ${ }^{16}$ The free absorbance of

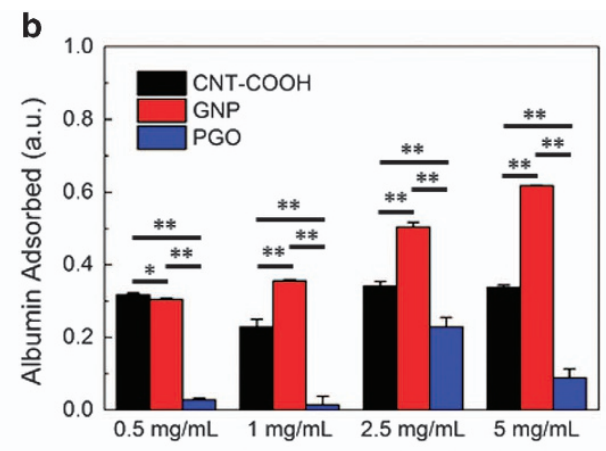

Albumin Concentration $(\mathrm{mg} / \mathrm{mL})$

d
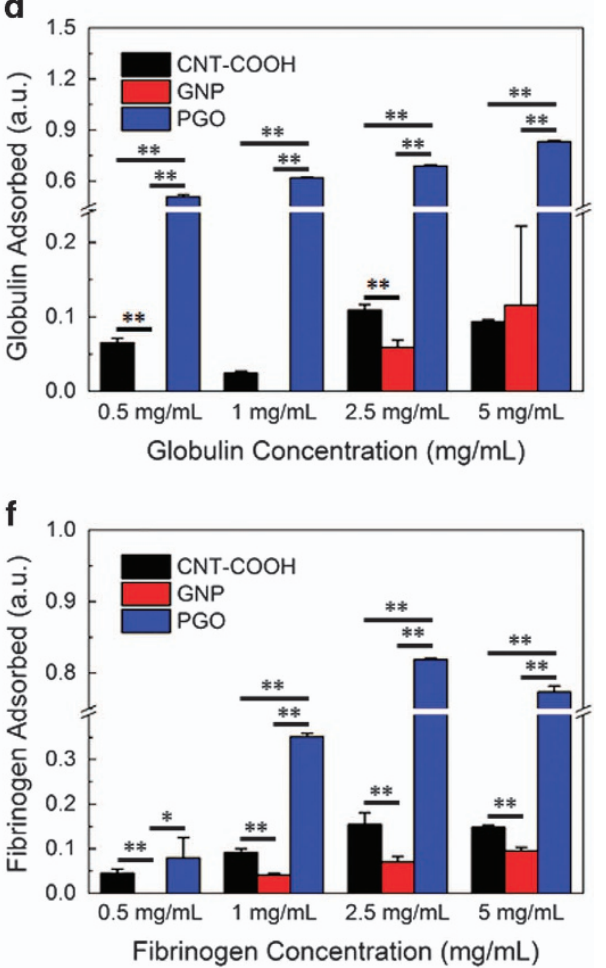

Figure 4 Blood plasma protein adsorption on carbon nanomaterials. Adsorption of plasma proteins: (a, b) albumin, (c, d) globulin and (e, f) fibrinogen on different carbon nanomaterials. (a, c, e) Adsorption of plasma proteins: (a) albumin, (c) globulin and (e) fibrinogen, on carbon nanomaterials as a function of protein concentrations. The Langmuir-Freundlich adsorption isotherm was used to model the obtained data, showing the saturation of protein adsorption on carbon nanomaterials. (b, d, f) Relative comparison of the adsorption of: (b) albumin, (d) globulin and (f) fibrinogen, on various carbon nanomaterials. For particular samples under comparison, statistically significant differences for $P<0.05$ and 0.01 were represented by * and **, respectively. The two-tailed Student's $t$-test was used for all statistical analyses. 
albumin, globulin and fibrinogen at numerous concentrations was first obtained at the specific wavelength of $280 \mathrm{~nm}$ (Supplementary Figure $\mathrm{S} 1 \mathrm{a}-\mathrm{c})$. From the calibration data, we noted a linear relationship between protein absorbance at $280 \mathrm{~nm}$ and protein concentration. The adsorption of specific plasma proteins on carbon nanomaterials was subsequently evaluated based on the plasma protein absorbance after incubations with carbon nanomaterials. For all plasma proteins, with an increasing protein concentration, a corresponding increase towards saturation in the protein adsorption on nanomaterials could be observed (Figures 4a,c and e). Nevertheless, we noted distinct protein adsorption behaviors on different carbon nanomaterials depending on the individual proteins. For albumin concentrations between 1 and $5 \mathrm{mg} \mathrm{ml}^{-1}$, the highest loading capacity for albumin was displayed by GNP, followed by CNT-COOH and PGO (Figures 4a and b). The higher adsorption of albumin on GNP than on CNT-COOH and PGO suggests that the associations between albumin and carbon nanomaterials might be strongly dependent on hydrophobic interactions. This is because the hydrophobic amino acids of albumin contain aromatic rings and these might adsorb on the carbon nanomaterial surface via $\pi-\pi$ stacking. As such, as compared to CNT-COOH and PGO, the hydrophobic GNP might be able to accommodate a higher amount of albumin based on hydrophobic interactions. Contrastingly, for all concentrations, the adsorption of albumin was significantly lower on PGO than on CNT-COOH and GNP.

Interestingly, a reverse trend was observed from the adsorption characteristics of globulin and fibrinogen on carbon nanomaterials (Figures 4c-f). More clearly, across all globulin and fibrinogen concentrations examined, PGO displayed the highest loading capacity for both proteins while those of CNT-COOH and GNP were fairly similar. The lower adsorption of fibrinogen on CNT-COOH and GNP (Figures $4 \mathrm{e}$ and $\mathrm{f}$ ) might be due to the large size of fibrinogen in relation to CNT-COOH and GNP which limited the ability of the molecule to interact with the two carbon nanomaterials. In addition, because of their long cord-like structure and high molecular weight, fibrinogen molecule tends to intertwine and form aggregates. As such, fibrinogen adsorption might require higher surface area as shown through the higher adsorption capacity of fibrinogen on PGO than on $\mathrm{CNT}-\mathrm{COOH}$ and GNP for the same fibrinogen concentration. It is also important to highlight that PGO possessed more oxygenated functional groups on its surface than CNT-COOH and GNP. The lower adsorption of albumin but the higher adsorption of globulin and fibrinogen on PGO signifies the pronounced influence of the oxygenated group density of carbon nanomaterials on plasma protein adsorption. Additionally, it is intriguing to note that for all globulin and fibrinogen concentrations, $1 \mathrm{D} \mathrm{CNT}-\mathrm{COOH}$ and 2D GNP exhibited relatively similar protein loading capacities. This possibly suggests the minimal influence of the dimensionality, surface curvature and specific surface area of carbon nanomaterials in the adsorption of these two plasma proteins. In short, in light of the experimental findings on the adsorption capacity of plasma proteins, we inferred that all carbon nanomaterials interacted with the plasma proteins to a certain extent. Crucially, the amount of these proteins loaded on carbon nanomaterials might be significantly influenced by the particular types of plasma proteins interacting with the nanomaterials, the amount of oxygen functional groups on the surface of carbon nanomaterials and to a lesser extent, the dimensionality of carbon nanomaterials.
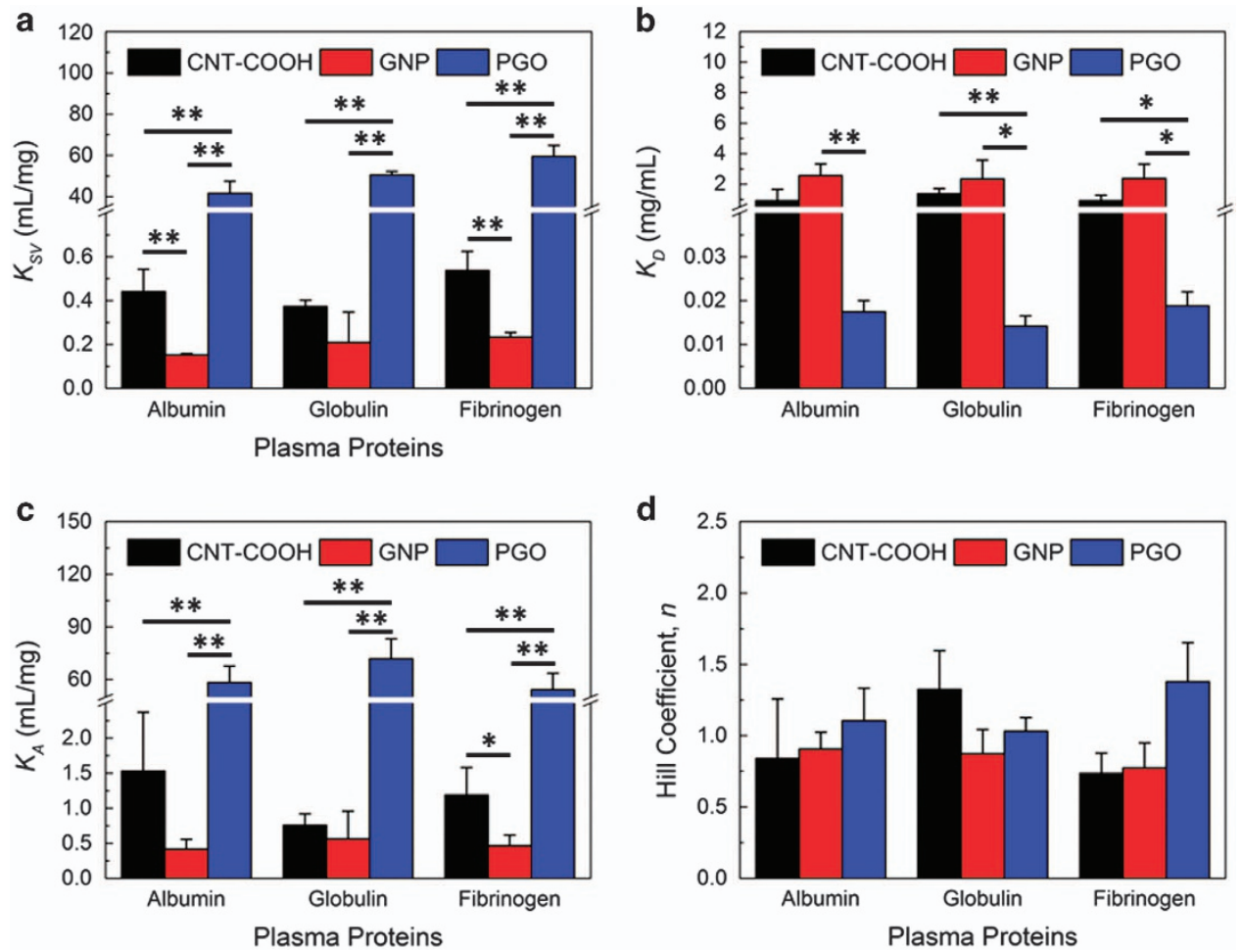

Figure 5 Fluorescence quenching efficiency, equilibrium binding association and binding cooperativity between carbon nanomaterials and plasma proteins. (a) Fluorescence quenching efficiency, as represented by $K_{\mathrm{Sv}}$, of carbon nanomaterials. (b-d) Nanomaterial-protein binding parameters: (b) Dissociation constant, $K_{\mathrm{D}}$, (c) Association constant, $K_{\mathrm{A}}$, and (d) Hill coefficient, $n$. For particular samples under comparison, statistically significant differences for $P<0.05$ and 0.01 were represented by * and $* *$, respectively. The two-tailed Student's $t$-test was used for all statistical analyses. 


\section{Fluorescence quenching and binding of plasma proteins}

In addition to protein adsorption, the quenching of the intrinsic fluorescence of plasma proteins in the presence of carbon nanomaterials was investigated (Supplementary Figure S2). Generally, proteins comprise essential aromatic amino acids, such as phenylalanine, tyrosine and tryptophan. These chromophore residues serve as the intrinsic fluorescent probes of plasma proteins and are highly sensitive to the local microenvironment. As such, variations in the emission characteristics of the active fluorescence emitters may be used as a direct indication of the protein binding-induced changes in the local molecular environment. As the concentrations of carbon nanomaterials (for example, PGO) increased, quenching of the intrinsic fluorescence of plasma proteins occurred and their fluorescence emission decreased progressively (Supplementary Figures S2a-c). This indicates a decrease in the relative distance between the quenching agent carbon nanomaterials and the protein chromophore residues as well as the direct interactions between them as plasma proteins adsorbed onto carbon nanomaterials. Moreover, as the fluorescence quenching grew stronger due to an increase in the nanomaterial concentrations, it was highly likely that the amount of the amino acid residues accessible to the nanomaterial surface increased, leading to possible conformational or tertiary structural changes of plasma proteins.

On the basis of the fluorescence quenching data, the essential quantitative parameters describing the binding between carbon nanomaterials and plasma proteins, in particular, quenching efficiency, association constant and binding cooperativity, were derived (Figure 5). The profiles showing the protein emission intensity (that is, $I^{0} / I$ ) against the nanomaterial concentrations were first obtained. In general, $I^{0}$ and $I$ denote the maximum protein emission intensity in the absence and presence of carbon nanomaterials, respectively. Interestingly, we observed that in the presence of carbon nanomaterials (for example, PGO) with increasing concentrations, the fluorescence emission $I^{0} / I$ of albumin, globulin and fibrinogen exhibited an exponential profile (Supplementary Figures S3a-c). This indicates that the intrinsic fluorescence of the three proteins was quenched both statically via ground-state complex formation as well as dynamically through collisional process. Furthermore, as the fluorescence quenching at low nanomaterial concentrations is typically influenced by diffusive transport, we computed the relative kinetic efficiency of quenching, based on the standard non-equilibrium Stern-Volmer mathematical model, as illustrated in Equation (1) (insets of Supplementary Figures S3a-c). In this model, the protein fluorescence emission was assumed to be linearly proportional to the quenching agent concentration. By fitting the experimental data to the model, the Stern-Volmer quenching constants were derived (Figure 5a).

As represented by the Stern-Volmer constant, that is, $K_{\mathrm{SV}}$, for all plasma proteins, we observed that PGO exhibited the highest fluorescence quenching efficiency while those of GNP and CNT$\mathrm{COOH}$ were significantly lower. With all proteins, the $K_{\mathrm{SV}}$ values of PGO were over two orders of magnitude higher than those of CNT$\mathrm{COOH}$ and GNP. In other words, the quenching efficiency of PGO was approximately 100 and 200 times higher than those of GNP and $\mathrm{CNT}-\mathrm{COOH}$, respectively. This might be attributed to the structural differences of carbon nanomaterials. The flat surface of PGO facilitates $\pi-\pi$ stacking between the aromatic rings of the three proteins and the nanomaterial more easily as compared to the curved surface of CNT$\mathrm{COOH}$. Furthermore, with a higher density of pores and oxygenated groups, it was highly likely that PGO possessed more surface defects as compared to CNT-COOH and GNP and these surface defects might serve as protein binding sites, enhancing protein adsorption and fluorescence quenching efficiency.

It is also noteworthy that the fluorescence quenching efficiency of 1D CNT-COOH was much higher than that of 2D GNP despite the much larger surface area of GNP. Typically, the relative surface area of a quenching agent is known to be directly correlated to its fluorescence quenching efficiency. In other words, a quenching agent with a large relative surface area will induce a strong fluorescence quenching and consequently, will have a high quenching efficiency, and vice versa. This is because, with a larger surface area, a quenching agent can accommodate a higher number of proteins, resulting in a more efficient fluorescence quenching. In contrast, a smaller relative surface area will induce a less efficient fluorescence quenching as only a small amount of proteins can attach onto the surface of the fluorescence quencher. Nevertheless, in our study, the direct correlation between the relative surface area of the quenching agent and its quenching efficiency was not evident. Instead, our observations on the higher quenching efficiency of CNT-COOH than GNP suggest the greater influence of the oxygenated functionality density than the relative surface area of carbon nanomaterials in their interactions with plasma proteins.

We further probed the relative strength and cooperativity of the carbon nanomaterial-plasma protein interactions based on the fluorescence quenching titration data (Supplementary Figure S4 and Figure 5). Using non-linear curve fitting based on Hill equation, as described in Equations (2) and (3) (Supplementary Figures S4a-c), we quantified several necessary parameters illustrating these associations, that is, dissociation constant $K_{\mathrm{D}}$, association constant $K_{\mathrm{A}}$ and Hill coefficient $n$ (Figures $5 \mathrm{~b}-\mathrm{d}$ ). These quantitative parameters were extracted under the assumption that the interactions took place under equilibrium. Firstly, the equilibrium dissociation constants, $K_{\mathrm{D}}$, of the various nanomaterial-protein complexes were estimated (Figure $5 b$ ). For all plasma proteins, the GNP-protein complexes displayed a comparatively higher $K_{D}$ than the CNT-COOH-protein complexes while the dissociation constants of the $\mathrm{PGO}$-protein complexes were significantly lower than those of both CNT-COOH-protein and GNP-protein complexes. Specifically, the GNP-protein complexes exhibited the highest $K_{\mathrm{D}}$ while the PGO-protein complexes had the lowest $K_{\mathrm{D}}$.

Following the computation of $K_{\mathrm{D}}$, we quantified the equilibrium association constant, $K_{\mathrm{A}}$, of the different nanomaterial-protein complexes (Figure $5 \mathrm{c}$ ). As $K_{\mathrm{A}}$ is the reciprocal of $K_{\mathrm{D}}$, it was anticipated that the GNP-protein and PGO-protein complexes would exhibit the lowest and highest $K_{\mathrm{A}}$, respectively. This indicates that PGO possessed the strongest associations with the three plasma proteins while the weakest plasma protein associations could be found with GNP. All three plasma proteins, in other words, had significantly higher affinity to PGO than to either CNT-COOH or GNP, based on the one to two orders of magnitude higher $K_{\mathrm{A}}$ values of the PGO-protein complexes as compared to those of other nanomaterial-protein complexes. In fact, it is important to highlight that no single plasma protein exhibited consistently higher affinity to specific carbon nanomaterial. Each protein appeared to associate strongly to certain carbon nanomaterials but weakly to others. For example, the order of affinity of plasma proteins to PGO was globulin > albumin > fibrinogen while for CNT$\mathrm{COOH}$, the order was albumin $>$ fibrinogen $>$ globulin. This suggests that the biological influence induced by carbon nanomaterials on plasma proteins may be protein-specific and nanomaterial-dependent.

It is important to note that although the differences in $K_{\mathrm{D}}$ and $K_{\mathrm{A}}$ between the nanomaterial-protein complexes formed by PGO and other carbon nanomaterials were significantly large (Figures $5 \mathrm{~b}$ and c), 
similar degree of differences was not reflected in the plasma protein adsorption on PGO and other carbon nanomaterials (Figure 4). This is because the adsorption of plasma proteins on carbon nanomaterials reflects the relative amount of plasma proteins loaded on carbon nanomaterials at the specific measurement points. In contrast, the metrics $K_{\mathrm{D}}$ and $K_{\mathrm{A}}$ indicate equilibrium bindings between carbon nanomaterials and plasma proteins in which saturation of proteins on nanomaterial surfaces had occurred. Consequently, the difference in the observed trends might be attributed to the different timescale of the two processes. Also, it is noteworthy that there was a linear relationship between the fluorescence quenching efficiency (that is, $K_{S V}$ ) of carbon nanomaterials and the association constants (that is, $K_{\mathrm{A}}$ ) of the nanomaterial-protein complexes. Clearly, the obtained data on the nanomaterial-protein associations offer an insight into the protein fluorescence quenching efficiency of carbon nanomaterials. For instance, albumin, globulin and fibrinogen associated most weakly with GNP. Simultaneously, GNP displayed the lowest efficiency in quenching the protein fluorescence. In contrast, the strongest protein fluorescence quenching and association could be achieved with PGO. All these suggest that the fluorescence quenching efficiency (as represented by $K_{\mathrm{SV}}$ ) was highly dependent on the distance between the chromophore residues of plasma proteins and the carbon nanomaterial surface (as represented by $K_{\mathrm{A}}$ ).

To further investigate the binding associations between carbon nanomaterials and plasma proteins, the cooperative nature of the nanomaterial-protein binding was examined (Figure $5 \mathrm{~d}$ ). In fact, the binding associations between carbon nanomaterials and plasma proteins were expected to display a particular level of cooperativity as it was highly possible that the three proteins possessed numerous binding sites on these nanomaterials. To verify this, using non-linear curve fitting based on Equations (2) and (3), we obtained the Hill coefficient, $n$, which describes the binding cooperativity or the dependency degree of the interactions between numerous binding sites. In general, a Hill constant $n<1$ indicates a negative cooperativity. This means that the affinity, the association energy per protein molecule, and the resultant binding strength of a nanomaterialprotein complex decreases progressively with further protein adsorption on the nanomaterial surface. In contrast, with a positive cooperativity (that is, Hill constant $n>1$ ), as the amount of proteins adsorb onto the nanomaterial surface increases, the affinity and association strength between this nanomaterial and proteins increase correspondingly.

Based on the acquired data, we noted that, for albumin, the Hill coefficients of the three nanomaterial-albumin complexes were quite similar (that is, $n \sim 1$ ). Apparently, the nanomaterial-albumin interactions had relatively neutral binding cooperativity, with all Hill coefficients close to 1 , although the average Hill coefficient of the PGO-albumin complex was slightly higher than those of CNT$\mathrm{COOH}$-albumin and GNP-albumin complexes. In the case of globulin, the CNT-COOH-globulin complex exhibited a much higher binding cooperativity than the GNP-globulin and PGO-globulin complexes. More clearly, the CNT-COOH-globulin complex exhibited a strongly positive cooperative reaction and its Hill coefficient was much higher than those of the other two complexes. For fibrinogen, the binding cooperativity of the PGO-fibrinogen complex was the highest among the three nanomaterial-fibrinogen formations. Interestingly, both the CNT-COOH-fibrinogen and GNP-fibrinogen complexes displayed anti-cooperative bindings with negative cooperativity.

The binding cooperativity of the carbon nanomaterial-plasma protein complexes might also be subtly influenced by the oxygen functionalities, dimensionalities and surface areas of the carbon nanomaterials. Among the nanomaterial-protein complexes, with the exception of CNT-COOH-albumin, the other PGO- and CNT$\mathrm{COOH}$-protein formations (that is, $\mathrm{PGO}-$ albumin, $\mathrm{PGO}-$ globulin, PGO-fibrinogen and CNT-COOH-globulin complexes) exhibited relatively positive cooperative reactions and had higher binding cooperativity (that is, average $n>1$ ) (Figure 5d). From our nanomaterial characterization results, we understood that PGO and CNT$\mathrm{COOH}$ possessed higher density of oxygenated functional elements as compared to GNP (Figures 3a-d). At the same time, PGO had a higher surface area than $\mathrm{CNT}-\mathrm{COOH}$ and GNP (Figures 1a-c). As such, the positive cooperative reactions of these complexes could probably be ascribed to the higher density of oxygen functionalities present on both PGO and CNT-COOH. As revealed via FTIR, XPS and Raman characterization, a direct interdependency between the density of the oxygenated groups of carbon nanomaterials and their defect concentrations might exist. Again, these surface defects might serve as the binding sites of the plasma proteins. Consequently, the availability of several binding sites, probably in the form of surface defects, between the nanomaterials and proteins would induce progressive increase in their binding affinity and cooperative binding associations. On the other hand, all three GNP-protein formations consistently displayed a relatively anti-cooperative binding characteristic with negative cooperativity. It was highly likely that the physicochemical properties of GNP varied as the three plasma proteins continuously adsorbed on the GNP surface over time.

As a whole, on the basis of our experimental data, we rationalized the strong positive correlation of the density of the oxygenated functional elements, dimensionality, curvature and surface area of carbon nanomaterials, with the binding cooperativity of the resultant nanomaterial-plasma protein complexes. Supplementary Table S1 summarizes all binding parameters describing the association between carbon nanomaterials and plasma proteins, as derived from the fluorescence quenching titration data.

\section{Conformational stability of plasma proteins}

Depending on the specific types of proteins and the surface chemistry of nanomaterials, protein adsorption on nanomaterial surface may induce protein conformational change or secondary and/or tertiary structural denaturation. This conformational alteration may dictate the extent to which proteins are able to perform their regular functionalities and biological activities. As such, it is important to assess changes to the protein conformation upon the interactions between plasma proteins and carbon nanomaterials in greater detail. Through CD spectroscopy, the degree of structural denaturation of plasma proteins upon the protein-nanomaterial interactions was examined. In the absence and presence of carbon nanomaterials, we recorded the far UV CD spectra of plasma proteins from 200 to $260 \mathrm{~nm}$ (Figure 6). Both albumin and fibrinogen displayed distinct $\alpha$-helical secondary structure, in which two negative bands at the characteristic wavelengths of 208 and $222 \mathrm{~nm}$ could be observed from their CD spectra (Figures $6 \mathrm{a}-\mathrm{c}$ and $\mathrm{g}-\mathrm{i}$ ). On the other hand, globulin possessed $\beta$-sheet secondary structure and its CD spectrum manifested a negative band at roughly $216 \mathrm{~nm}$ (Figures $6 \mathrm{~d}-\mathrm{f}$ ).

Upon interactions with different carbon nanomaterials with increasing concentrations, we noted that the CD spectra of pure albumin and albumin-nanomaterial complexes coincided closely and the ellipticity of all spectra was largely unperturbed (Figures $6 a-c$ ). This suggests that there was no denaturation to the secondary structure of albumin and it was able to maintain conformational stability in the presence of various carbon nanomaterials. Similarly, for 
a

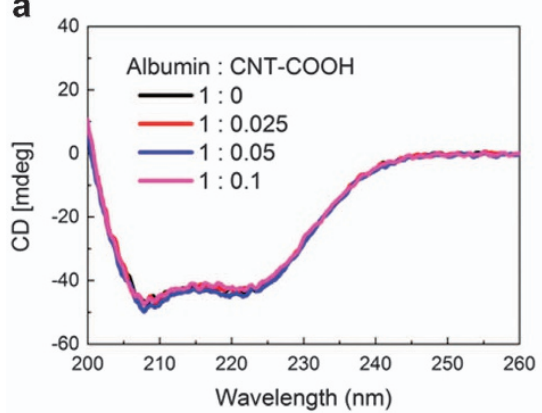

d

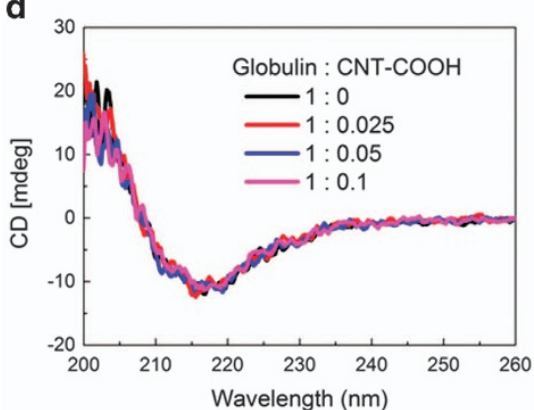

g

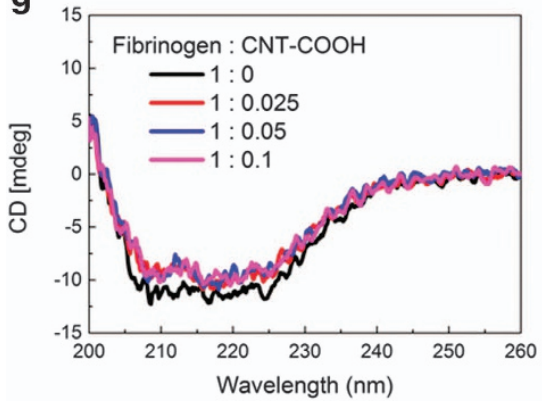

b

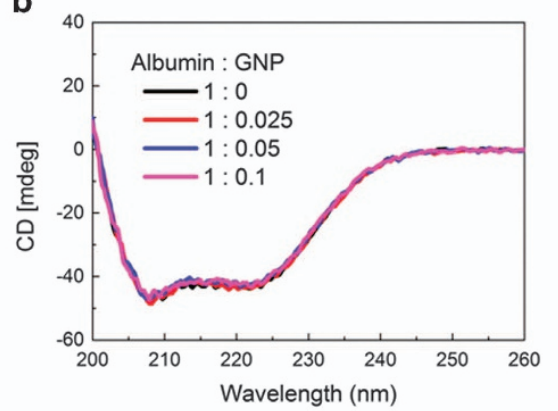

e

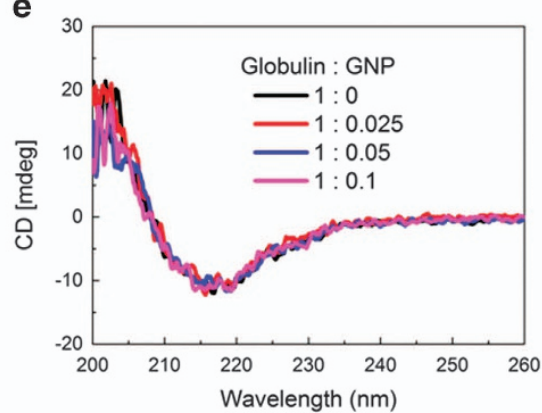

h

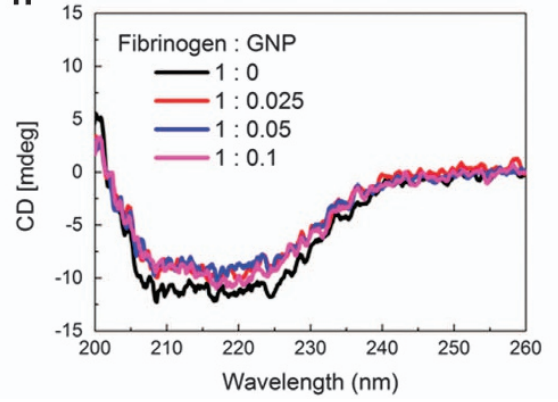

C

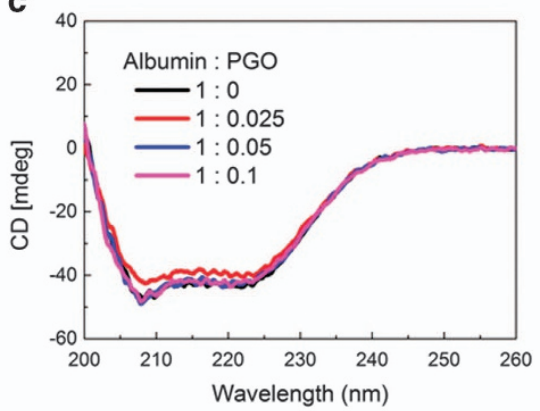

f

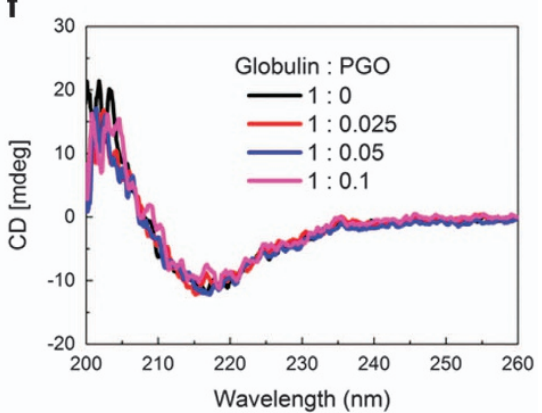

i

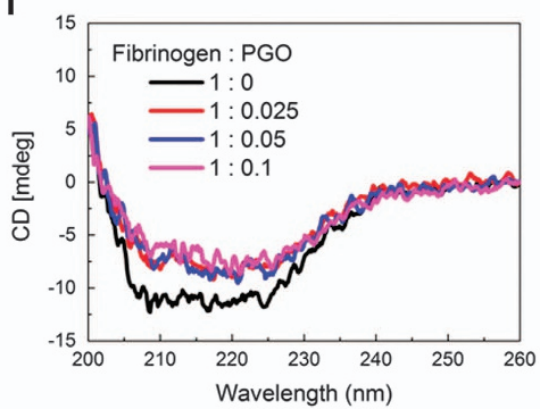

Figure 6 Secondary structural stability of plasma proteins in the presence of carbon nanomaterials with varying concentrations. CD spectra of (a-c) albumin, (d-f) globulin and (g-i) fibrinogen upon interactions with carbon nanomaterials, that is, CNT-COOH, GNP and PGO, with different concentrations. Albumin, globulin and fibrinogen had fixed concentrations of 200, 400 and $400 \mu \mathrm{g} \mathrm{ml}^{-1}$, respectively, and the protein-nanomaterial complexes had concentration ratios of 1:0.025, 1:0.05 and 1:0.1. CD, circular dichroism; CNT-COOH, COOH-functionalized carbon nanotube; GNP, graphene nanoplatelet; PGO, porous graphene oxide.

globulin, it was evident that all recorded CD spectra of pure globulin and globulin-nanomaterial complexes overlapped and there were negligible variations in their ellipticity (Figures 6d-f). Again, this indicates the conformational stability of globulin in the presence of CNT-COOH, GNP and PGO and that globulin was still able to retain its $\beta$-sheet secondary structure upon associations with these carbon nanomaterials.

Interestingly, for fibrinogen, we observed that upon conjugations with the three carbon nanomaterials, the resultant fibrinogen-nanomaterial complexes showed a decreased ellipticity in their CD spectra (Figures $6 \mathrm{~g}-\mathrm{i}$ ). The decrease in the ellipticity was apparent from the attenuation of the two $\alpha$-helical bands at 208 and $222 \mathrm{~nm}$. In fact, the negative band attenuations induced by CNT-COOH and GNP were roughly similar in magnitude irrespective of the concentrations of these two nanomaterials (Figures $6 \mathrm{~g}$ and $\mathrm{h}$ ). Nevertheless, despite the variations in ellipticity, importantly, the $\alpha$-helical secondary structure of fibrinogen was still highly preserved in the presence of $\mathrm{CNT}-\mathrm{COOH}$ and GNP with various concentrations. Contrastingly, this was not the case with PGO. Even at low concentrations of 10 and $20 \mu \mathrm{g} \mathrm{ml}^{-1}$, the $\alpha$-helical bands of fibrinogen were significantly attenuated by PGO (Figure 6i). In particular, the negative band at $208 \mathrm{~nm}$ started to diminish. Moreover, as the concentration of PGO increased to $40 \mu \mathrm{g} \mathrm{ml}^{-1}$, an enhanced attenuation of the $208 \mathrm{~nm}$ band as well as an overall reduction of the ellipticity of the CD spectrum could be observed. This suggests that PGO significantly disrupted the secondary structure and overall conformational stability of fibrinogen, resulting in the possible unraveling of $\alpha$-helix domains coupled with the denaturation of fibrinogen upon its binding to PGO. As a whole, based on all $\mathrm{CD}$ characterization data, we noted the oxygen functionality-dependent effect of carbon nanomaterials in affecting the secondary structure and overall conformation of fibrinogen upon the fibrinogen-nanomaterial interactions.

\section{CONCLUSIONS}

In summary, we have investigated the nano-bio interactions between blood plasma proteins and carbon nanomaterials to gain insights into the molecular hemocompatibility of these engineered nanomaterials. Carbon nanomaterials with different dimensionalities and oxygenated 
group densities (that is, 1D CNT-COOH and 2D GNP and PGO) and several major plasma proteins (that is, albumin, globulin and fibrinogen) were used in this study. In light of the obtained qualitative and quantitative parameters, we have established various aspects of the nanomaterial-plasma protein interactions, including protein adsorption, nanomaterial-protein equilibrium binding and protein conformational stability. Importantly, we have demonstrated that the interactions between carbon nanomaterials and plasma proteins might be strongly dependent on the oxygenated functional group density of the nanomaterials, particularly the amount of their $\mathrm{C}-\mathrm{O}$ and $\mathrm{C}=\mathrm{O}$ bonds, and, to a certain extent, the nanomaterial dimensionality, surface curvature and surface area. We foresee that this work will further unravel the nano-bio interactions between carbon nanomaterials and blood plasma proteins and offer a strong basis for enhanced design and use of different carbon nanomaterials for numerous bioapplications.

\section{CONFLICT OF INTEREST}

The authors declare no conflict of interest.

\section{ACKNOWLEDGEMENTS}

Kenry and AG would like to acknowledge the NUS Graduate School for Integrative Sciences and Engineering Scholarship and Whitaker International Fellowship, respectively. KPL acknowledges the support of NRF investigator award: 'Graphene Oxide-A new case of catalytic, ionic, and molecular sieving materials [R-143-000-610-281]'. This research was supported by the National Research Foundation, Prime Minister's Office, Singapore under its mediumsized centre program, Centre for Advanced 2D Materials and its Research Centre of Excellence, Mechanobiology Institute, as well as the MechanoBioEngineering Laboratory of the Department of Biomedical Engineering of the National University of Singapore. The authors would like to acknowledge Ms Fengmei Li from the Department of Chemical and Biomolecular Engineering, NUS, for rendering her assistance in the FTIR and CD spectroscopy measurements.

1 Thostenson, E. T., Ren, Z \& Chou, T-W Advances in the science and technology of carbon nanotubes and their composites: a review. Compos Sci. Technol. 61, 1899-1912 (2001).

2 De Volder, M. F. L., Tawfick, S. H., Baughman, R. H. \& Hart, A. J. Carbon nanotubes: present and future commercial applications. Science 339, 535-539 (2013).

3 Zhang, Q, Huang, J-Q, Qian, W-Z, Zhang, Y-Y \& Wei, F The road for nanomaterials industry: a review of carbon nanotube production, post-treatment, and bulk applications for composites and energy storage. Small 9, 1237-1265 (2013).

4 Geim, A. K. Graphene: status and prospects. Science 324, 1530-1534 (2009).

5 Allen, M. J., Tung, V. C. \& Kaner, R. B. Honeycomb carbon: a review of graphene. Chem. Rev. 110, 132-145 (2010).

6 Singh, V, Joung, D, Zhai, L, Das, S, Khondaker, S. I. \& Seal, S Graphene based materials: past, present and future. Prog. Mater. Sci. 56, 1178-1271 (2011).

7 Novoselov, K. S., Falko, V. I., Colombo, L, Gellert, P. R., Schwab, M. G. \& Kim, K A roadmap for graphene. Nature 490, 192-200 (2012).

8 Lu, F, Gu, L, Meziani, M. J., Wang, X, Luo, P. G., Veca, L. M., Cao, L \& Sun, Y-P Advances in bioapplications of carbon nanotubes. Adv. Mater. 21, 139-152 (2009).

9 Liu, Z, Tabakman, S, Welsher, K \& Dai, H Carbon nanotubes in biology and medicine: in vitro and in vivo detection, imaging and drug delivery. Nano Res. 2, 85-120 (2009).

10 Jin, L, Yang, K, Yao, K, Zhang, S, Tao, H, Lee, S-T, Liu, Z \& Peng, R Functionalized graphene oxide in enzyme engineering: a selective modulator for enzyme activity and thermostability. ACS Nano 6, 4864-4875 (2012).

11 Chung, C, Kim, Y-K, Shin, D, Ryoo, S-R, Hong, B. H. \& Min, D-H Biomedical applications of graphene and graphene oxide. Acc. Chem. Res. 46, 2211-2224 (2013)
12 Tan, X, Feng, L, Zhang, J, Yang, K, Zhang, S, Liu, Z \& Peng, R Functionalization of graphene oxide generates a unique interface for selective serum protein interactions. ACS Appl. Mater. Interfaces 5, 1370-1377 (2013).

13 Lee, W. C., Lim, C. H., Kenry, Su, C, Loh, K. P. \& Lim, C. T. Cell-assembled graphene biocomposite for enhanced chondrogenic differentiation. Small 11, 963-969 (2015).

14 Ng, AM. H., Kenry, Teck Lim, C, Low, H. Y. \& Loh, K. P. Highly sensitive reduced graphene oxide microelectrode array sensor. Biosens. Bioelectron. 65, 265-273 (2015)

15 Kenry, Chaudhuri, P. K., Loh, K. P. \& Lim, C. T. Selective accelerated proliferation of malignant breast cancer cells on planar graphene oxide films. ACS Nano 10, 3424-3434 (2016).

16 Kenry, Loh, K. P. \& Lim, C. T. Molecular hemocompatibility of graphene oxide and its implication for antithrombotic applications. Small 11, 5105-5117 (2015).

17 Kenry, Loh, K. P. \& Lim, C. T. Molecular interactions of graphene oxide with human blood plasma proteins. Nanoscale 8, 9425-9441 (2016).

18 Kenry, Loh, K. P. \& Lim, C. T. Selective concentration-dependent manipulation of intrinsic fluorescence of plasma proteins by graphene oxide nanosheets. RSC Adv. 6 , 46558-46566 (2016).

19 Kenry, Yeo, J. C., Yu, J, Shang, M, Loh, K. P. \& Lim, C. T. Highly flexible graphene oxide nanosuspension liquid-based microfluidic tactile sensor. Small 12, 1593-1604 (2016)

20 Sanchez, V. C., Jachak, A, Hurt, R. H. \& Kane, A. B. Biological interactions of graphene-family nanomaterials: an interdisciplinary review. Chem. Res. Toxicol. 25 , 15-34 (2012).

21 Liu, Y, Zhao, Y, Sun, B \& Chen, C Understanding the toxicity of carbon nanotubes. Acc. Chem. Res. 46, 702-713 (2013).

22 Guo, X \& Mei, N Assessment of the toxic potential of graphene family nanomaterials. J. Food Drug Anal. 22, 105-115 (2014).

23 Seabra, A. B., Paula, A. J., de Lima, R, Alves, O. L. \& Durán, N Nanotoxicity of graphene and graphene oxide. Chem. Res. Toxicol. 27, 159-168 (2014).

24 Lundqvist, M, Stigler, J, Elia, G, Lynch, I, Cedervall, T \& Dawson, K. A. Nanoparticle size and surface properties determine the protein corona with possible implications for biological impacts. Proc. Natl. Acad. Sci. USA 105, 14265-14270 (2008).

25 Verma, A \& Stellacci, F Effect of surface properties on nanoparticle-cell interactions. Small 6, 12-21 (2010).

26 Saptarshi, S. R., Duschl, A \& Lopata, A. L. Interaction of nanoparticles with proteins: relation to bio-reactivity of the nanoparticle. J. Nanobiotechnol. 11, 1-12 (2013).

27 Lynch, I \& Dawson, K. A. Protein-nanoparticle interactions. Nano Today 3, 40-47 (2008).

28 Nel, A. E., Madler, L, Velegol, D, Xia, T, Hoek, EM. V., Somasundaran, P, Klaessig, F, Castranova, V \& Thompson, M Understanding biophysicochemical interactions at the nano-bio interface. Nat. Mater. 8, 543-557 (2009).

29 Shemetov, A. A., Nabiev, I \& Sukhanova, A Molecular interaction of proteins and peptides with nanoparticles. ACS Nano 6, 4585-4602 (2012).

30 Lacerda, S. H. D. P., Park, J. J., Meuse, C, Pristinski, D, Becker, M. L., Karim, A \& Douglas, J. F. Interaction of gold nanoparticles with common human blood proteins. ACS Nano 4, 365-379 (2010).

$31 \mathrm{Ge}, \mathrm{C}, \mathrm{Du}, \mathrm{J}$, Zhao, L, Wang, L, Liu, Y, Li, D, Yang, Y, Zhou, R, Zhao, Y, Chai, Z \& Chen, C Binding of blood proteins to carbon nanotubes reduces cytotoxicity. Proc. Nat/. Acad. Sci. USA 108, 16968-16973 (2011).

32 Meng, J, Song, L, Xu, H, Kong, H, Wang, C, Guo, X \& Xie, S Effects of single-walled carbon nanotubes on the functions of plasma proteins and potentials in vascular prostheses. Nanomed.: Nanotechnol. Biol. Med. 1 , 136-142 (2005).

33 Su, C, Acik, M, Takai, K, Lu, J, Hao, S-j, Zheng, Y, Wu, P, Bao, Q, Enoki, T, Chabal, Y. J. \& Ping Loh, K Probing the catalytic activity of porous graphene oxide and the origin of this behaviour. Nat. Commun. 3, 1298 (2012).

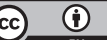

This work is licensed under a Creative Commons Attribution 4.0 International License. The images or other third party material in this article are included in the article's Creative Commons license, unless indicated otherwise in the credit line; if the material is not included under the Creative Commons license, users will need to obtain permission from the license holder to reproduce the material. To view a copy of this license, visit http:// creativecommons.org/licenses/by/4.0/

(C) The Author(s) 2017

Supplementary Information accompanies the paper on the NPG Asia Materials website (http://www.nature.com/am) 